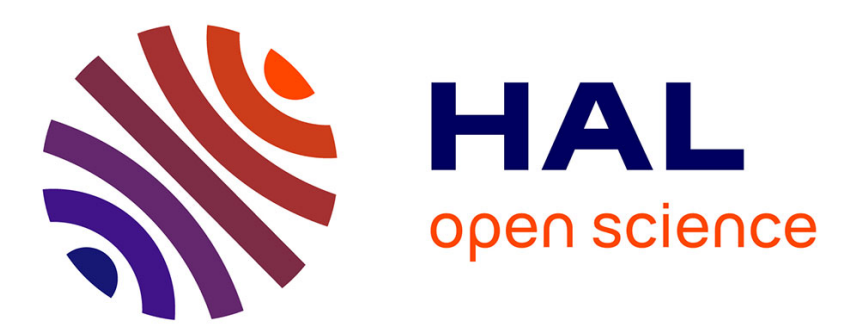

\title{
Storage and injection of charged particles in an electrostatic trap
}

Chun-Sing O., H.A. Schuessler

\section{To cite this version:}

Chun-Sing O., H.A. Schuessler. Storage and injection of charged particles in an electrostatic trap. Revue de Physique Appliquée, 1981, 16 (8), pp.441-445. 10.1051/rphysap:01981001608044100 . jpa00244938

\section{HAL Id: jpa-00244938 https://hal.science/jpa-00244938}

Submitted on 1 Jan 1981

HAL is a multi-disciplinary open access archive for the deposit and dissemination of scientific research documents, whether they are published or not. The documents may come from teaching and research institutions in France or abroad, or from public or private research centers.
L'archive ouverte pluridisciplinaire HAL, est destinée au dépôt et à la diffusion de documents scientifiques de niveau recherche, publiés ou non, émanant des établissements d'enseignement et de recherche français ou étrangers, des laboratoires publics ou privés. 


\title{
Storage and injection of charged particles in an electrostatic trap
}

\author{
Chun-Sing $\mathrm{O}$ and H. A. Schuessler \\ Department of Physics, Texas A \& M University, College Station, Texas 77843, U.S.A.
}

(Reçu le 23 avril 1981, accepté le 14 mai 1981)

\begin{abstract}
Résumé. - Le confinement des ions à l'intérieur d'une cage purement électrostatique est étudié. La cage est composée de deux cylindres concentriques maintenus à une différence de potentiel et de deux couvercles plans isolés. Si la longueur de la cage est longue comparée à la dimension radiale, le potentiel créé dans la région intérieure est logarithmique. Le domaine de confinement est déterminé en comparant les déplacements radiaux d'un ion à l'apogée et au périgée aux rayons des cylindres. L'effet d'un champ magnétique uniforme superposé le long de la cage est considéré et le stockage des ions injectés de l'extérieur est étudié numériquement. La cage électrostatique des ions est plus intéressante lorsqu'il faut piéger des particules chargées indépendamment du rapport charge/ masse.
\end{abstract}

\begin{abstract}
Ion confinement in an purely electrostatic ion trap is investigated. The trap consists of two concentric cylinders maintained at a potential difference and two electrically insulated end plates. If the length of the trap is long compared with the radial dimension of the trap, the radial potential distribution is practically logarithmic. The domain of ion confinement is derived by comparing the radial displacements at the apogee and the perigee of an ion orbit with the radii of the cylinders. The effect of a magnetic field superimposed along the trap axis is considered. In addition trapping of externally injected ions is studied numerically. The electrostatic trap is used to advantage, when it is required to store charge particles independent of their charge to mass ratio.
\end{abstract}

1. Introduction. - The confinement of charged particles in three dimensions has found applications in a widening range of fields of physics. Most notably ion storage techniques were used for the precision spectroscopy of ions [1] and charged particles [2], in studies of ion-molecule reactions [3], in mass spectrometry [4], and in ultra high vacuum technology [5]. Miniature ion traps are also of importance in nuclear spectroscopy, where it is advantageous to confine minute samples of radioactive isotopes in a small volume. Various ion traps were developed, and may be grouped into two categories according to the form of the storage potentials : those that use a quadrupole potential and those that use a logarithmic potential distribution. While traps of the first category have been widely employed, those of the second have not. Quadrupole potentials are used in the rf trap [6], the Penning trap [7], and the combined trap [8]. These types of traps have discrete ion oscillation frequencies and allow chargeto-mass ratio selective ion storage. On the other hand a logarithmic trapping potential is present in the purely electrostatic trap $[9,10]$. Here a broad continuum of ion oscillation frequencies occurs and the storage properties are independent of the ion charge- to-mass ratio. In experimental situations, where it is desirable to trap ions of very different charge-to-mass ratios, the purely electrostatic trap is used to advantage: The electric fields to operate this trap are very small and produce a negligible Stark splitting. An additional magnetic field can be applied as needed for spectroscopy, and is not required for trapping. Most important however rf heating [11], which is the major ion loss mechanism in rf traps, is not present in the electrostatic trap and therefore the requirement of operation in ultra-high vacuum is less stringent. The electrostatic trap has so far been used to determine the hyperfine structure splitting of the metastable $2 \mathrm{~S}$ state [12] of ${ }^{3} \mathrm{He}^{+}$and recently to investigate electron capture processes [13] of highly charged ions.

Our interest in the electrostatic trap was aroused by its capacity for simultaneously trapping ions of very different charge-to-mass ratios, but also by its simple geometry and ease of operation. In connection with our previous work $[14,15]$ on various other ion trap devices, the possible application of the electrostatic trap for the storage of injected ions is of interest. If ions can be caught in flight after they are produced at low energies [16] in a heavy ion collision, or after 
suitable deceleration as they emerge from an accelerator, novel types of experiments will become possible. In particular the sensitivity and resolution of on-line spectroscopy of short-lived isotopes and highly charged ions will be increased by orders of magnitude, since fluorescence from a singly stored ion [17] and laser side-band cooling [17, 18] below $1 \mathrm{~K}$ have already been demonstrated.

The paper is divided into six sections. Section 2 gives a description of the ion motion in a logarithmic potential. The domain of ion confinement is derived in section 3. The effect of an external magnetic field on the confinement properties is discussed in section 4. Section 5 presents a treatment of injection of ions generated externally. The results of the calculations are summarized in section 6 .

2. Geometry of the trapping fields and theory of operation. - The geometry of the electrostatic ion trap is simple. It consists of two coaxial cylinders with electrically isolated plates on each end. The inner cylinder usually has the form of a thin wire. The complete configuration is shown in figure $1 a$. To trap positively charged ions a constant potential difference is maintained between the two coaxial cylinders with the inner cylinder electrically more negative to produce an attractive radial electric field. In addition a potential positive with respect to the inner cylinder is maintained at the end plates to restrict the ion motion along the axial direction. The ion trajectory is the superposition of a central force motion in the radial direction and an oscillating motion along the cylinder axis. Ideally for coaxial cylinders of infinite length the potential distribution in the space inbetween is simply logarithmic. For an electrostatic ion trap of finite length, this is not necessarily true. Figures $1 b, 1 c$ and $1 d$ show the potential distribution, obtained numerically for one quadrant of a cross section, for electrostatic traps of different lengths. In the example shown the inner cylinder potential is $-10 \mathrm{~V}$, and both end plates and

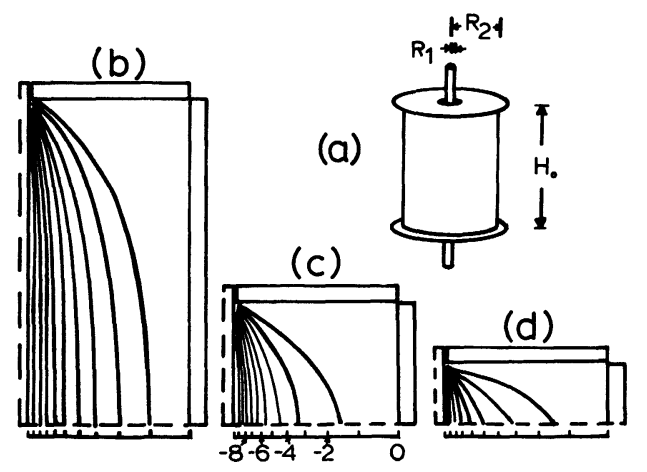

Fig. 1. - Configuration of the electrostatic trap and internal potential distribution, when the inner cylinder is at $-10 \mathrm{~V}$ and both end plates and the outer cylinder are grounded. Only one quadrant of the longitudinal cross section is shown. (a) Trap configuration. The ratio $H_{0} / 2 R_{2}$ is $1.8,0.7$, and 0.35 for $(b),(c)$, and $(d)$, respectively. The accompanying scale represents the equipotential positions for a logarithmic potential distribution. the outer cylinder are grounded. For very short axial lengths the radial potential distribution is quite different from a logarithmic case. However, when the trap axial length is more than one and a half times the diameter of the outer cylinder, figure $1 b$, the radial potential distribution at the equatorial plane of the ion trap is well represented by a logarithmic potential distribution. In addition the equatorial plane potential distribution is not seriously affected by the potential level at the end plates. On the other hand the axial potential distribution cannot be represented analytically. In general for a long electrostatic trap the ion inside sees a negligible axial field lengthwise until it gets close to either end where a repulsive axial field occurs. The ion axial motion is basically a back and forth drift at nearly constant speed except when the ion is very close to the end plates where it is reflected by a steep potential barrier. The radial motion of ions in the central portion of the trap can be described using the logarithmic potential well model. The radial distributions of the potential and the attractive electric field strength for a logarithmic potential well are depicted in figure 2. Also shown are the corresponding quantities for a harmonic potential well, which serves as an approximation to a quadrupole ion trap. An important difference is that the attractive electric field strength is proportional to the inverse of the radial displacement in a logarithmic potential well in contrast to the proportional relationship for the harmonic potential well. The ion motion in a radial logarithmic potential well has been studied earlier [10]. The ion is either accelerated or decelerated radially depending on the relative magnitudes of the attractive electric force and the centrifugal force due to its angular momentum at each instance

$$
\ddot{r}=\frac{L^{2}}{m^{2} r^{3}}-\frac{q V}{m \ln \left(R_{2} / R_{1}\right)} \frac{1}{r} .
$$

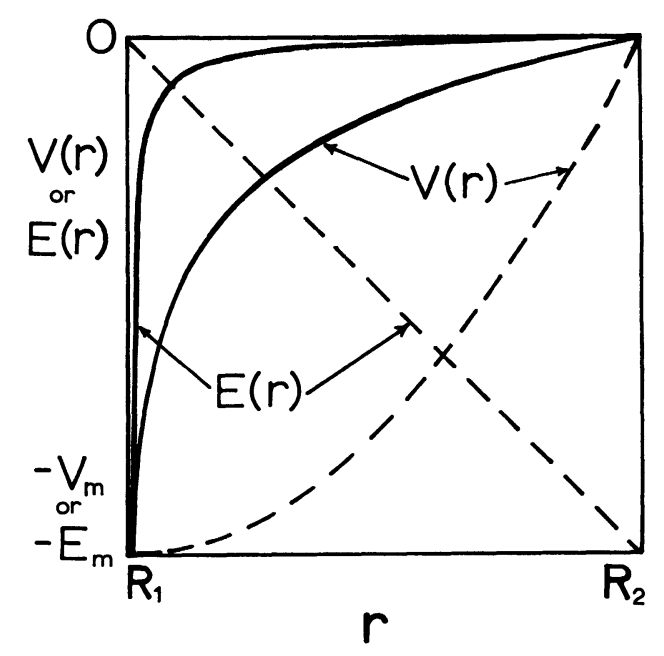

Fig. 2. - Radial distributions of the potential and the electric field strength for a logarithmic potential well (solid lines) are a harmonic potential well (dashed lines). 
Here $L$ is the constant angular momentum, $q / m$ is the charge-to-mass ratio of the ion, $V$ is the potential difference between the axial cylinders and $R_{1}$ and $R_{2}$ are the radii of the inner and the outer cylinders, respectively. Some typical ion trajectories are shown in figure 3 where the ratio of the potential energy of an ion and its initial kinetic energy, $P=|q V| / T_{0}$, is varied. When the value of $P$ is very small, the radial potential well does not generate enough attractive force to compensate for the centrifugal force. The ion will spin out to hit the outer cylinder, as shown in figure $3 a$. When the value of $P$ is larger, the radial electric attractive force is increased so that the ion orbit does not intersect the outer cylinder. At even higher $P$ values, the ion has a similar orbit shape but is pulled more closely to the inner cylinder. Such trapping orbits are depicted in figures $3 b$ and $3 c$. As $P$ is further increased to exceed a certain value, which, for the present example, is of the order of 100 , the ion orbit becomes elongated. At very high $P$ values the ion orbit consists of long and narrow loops which lead to the ion orbit again intersecting the outer cylinder, as shown in figure $3 d$. For the example shown the values of $P$ must be within the range from 4 to 500 for the ion orbit to be clear both cylinders. For an ion created inside of the trap by electron impact ionization of photoionization, its initial kinetic energy is typically the thermal energy of its parent neutral particle, i.e. about $0.026 \mathrm{eV}$ at room temperature. Therefore the applied potential difference between the two cylinders should not be less than $0.1 \mathrm{~V}$ or exceed $13 \mathrm{~V}$ for such ions to be trapped.

3. Domain of ion confinement. - For an ion to be confined the radial displacements at the apogee and perigee of the orbit must be smaller than $R_{2}$ and larger than $R_{1}$, respectively. Otherwise the ion hits either the inner or the outer cylinder and is lost. The following scheme has been devised to determine which orbits are bound in the electrostatic trap. From a knowledge of the initial position and the angular momentum the confinement property of the electrostatic trap for an internally created ion is obtained for each trap size and applied potential difference. In this procedure the domain of confinement is presented as a $\theta$ versus $r_{0}$ plot, where $\theta$ is the angle of the initial velocity with respect to a radius vector and $r_{0}$ is the initial ion position. For a given trap with $R_{2}=1.25 \mathrm{~cm}$ and $R_{1}=0.01 \mathrm{~cm}$ the domains of stability are depicted in figure $4 a$ for different values of the potential to initial kinetic energy ratio $P$. The initial condition for trajectories in figure 3 is shown as the small circle. When $P=1$, no domain of confinement exists. No ion can be confined in this case because the radial attraction force is always too weak to compensate for the centrifugal force. The initial condition circle is inside the corresponding domains for $P=10$ and 100. Such an ion can be confined. But for $P=10$ an ion created with $r_{0} / R_{2}>0.7$ will have too large
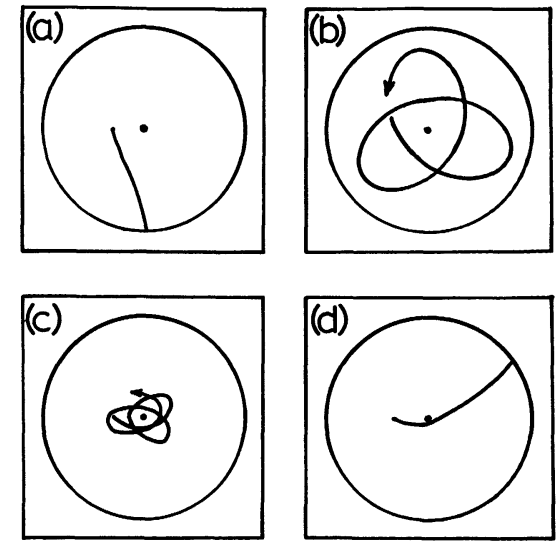

Fig. 3. - Typical ion trajectories on a radial plane inside the electrostatic trap. The initial ion position is $r_{0} / R_{2}=0.3$ and $\theta=45^{\circ}$. The values of $P=|q V| / T_{0}$ are $1,10,100$, and 1000 for $(a),(b),(c)$, and $(d)$, respectively.
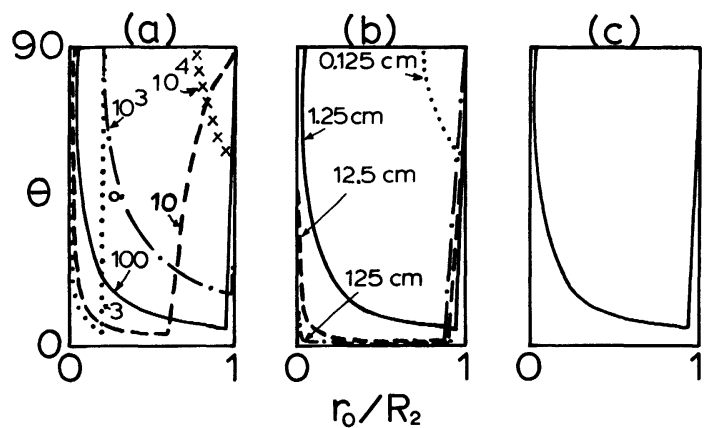

Fig. 4. - Domain of ion confinement. (a) Electrostatic trap with $R_{2}=1.25 \mathrm{~cm}$ and $R_{1}=0.01 \mathrm{~cm}$ but different values of $P$. (b) Electrostatic trap with different values of $R_{2}$ but the same values of $R_{1}=0.01 \mathrm{~cm}$ and $P=100$. (c) Electrostatic trap with different values of $P, R_{1}$, and $R_{2}$ but the same values of $R_{2} / R_{1}=125$ and $P_{R}=P / \ln \left(R_{2} / R_{1}\right)=20.7$.

an angular momentum to be confined. On the other hand when $P=1000$ the corresponding domain of confinement does not cover the initial condition circle which means that the ion will not be confined. When $P=10^{4}$ the radial attraction is so large that the ion angular momentum must also be large for the orbit to clear the inner cylinder. This fact is reflected by the presence of the domain of stability on the upper right hand corner of the $\theta$ versus $r_{0} / R_{2}$ plot, where it represents high values of $\theta$ and $r_{0} / R_{2}$. If the probability of ion creation is the same at each point inside the electrostatic trap and the created ion moves with equal probability in different directions, then a larger domain of ion confinement implied that more ions can be confined. Among the five cases depicted in figure $4 a$, the value of $P=100$ is the most favourable for ion confinement. The effect of changing the size of the outer cylinder but maintaining the same energy ratio with $P=100$ on the domain of stability is shown in figure $4 b$. The larger the outer cylinder the better is the confinement property. This is due to the larger angular momentum an ion possesses, when it is created at the same relative initial position $r_{0} / R_{2}$. 
Figure $4 c$ depicts the effect of scaling the electrostatic trap but maintaining a new quantity $P_{R}=P / \ln \left(R_{2} / R_{1}\right)$ constant. Hereby the values of $R_{2}$ and $R_{1}$ are changed over five orders of magnitude, but their ratio is left the same. When using the scaled energy ratio $P_{R}$ no variation of the domain of confinement is present.

4. Ion storage in the presence of a superimposed external magnetic field. - The effect of a homogeneous magnetic field applied parallel to the trap axis on the ion orbits is discussed next. Such a magnetic field is sometimes necessary for Zeeman spectroscopy or polarized ion confinement experiments, but not for ion storage. The presence of the superimposed magnetic field of field strength $H$ introduces a precession of the ion motion [7]. The ion acquires a cyclotron frequency $\omega_{\mathrm{c}}=q H / m$ and follows its original central force orbit in the precessing coordinate frame. When transferred back to the laboratory frame of reference, the angular momentum $L$ is not constant but instead a new angular momentum $\mathbf{L}^{\prime}=\mathbf{L}+m r^{2} \omega_{c} / 2$ becomes the constant of motion. Depending on the relative orientation of the vectors $\mathbf{L}$ and $\omega_{c}$ the new angular momentum $\mathbf{L}^{\prime}$ can be either larger or smaller than the original angular momentum $L$. The equation of motion in the radial direction is changed to

$$
\ddot{r}=\frac{L^{\prime 2}}{m^{2} r^{3}}-\frac{\omega_{\mathrm{c}}^{2}}{4} r-\frac{q V}{m \ln \left(R_{2} / R_{1}\right)} \frac{1}{r} .
$$

In the low magnetic field case, the motion is very similar to the zero field situation except that the domains of ion confinement for the two different values of $L^{\prime}$ are shifted slightly in opposite directions. On the other hand in the strong magnetic field case the ions simply follows circular orbits around the field lines. In the case of an intermediate field the relative orientation of the two vectors $L$ and $\omega_{c}$ is important because of the different resultant values of $L^{\prime}$. A typical case when the values of $\omega_{\mathrm{c}} / 2$ and the initial rate of revolution are about the same is depicted in figure 5 . Because of the fact that $L^{\prime}$ is approximately $2 L$ or 0 when $\mathbf{L}$ and $\omega_{c}$ are parallel or antiparallel to each other, respectively, the ion orbits take on very different shapes. However under typical experimental conditions the value of $H$ is normally very low and its effect on the ion motion can be neglected.
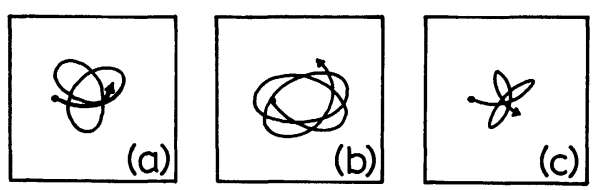

Fig. 5. - Typical ion orbit in the presence of an axial magnetic field. $(a)$ is the case of zero magnetic field. $(b)$ and $(c)$ are for the cases where $\mathbf{L}$ and $\omega_{c}$ are parallel and antiparallel to each other, respectively. The magnetic field strength is of a value so that $m r_{0}^{2} \omega_{c} / 2$ is approximately equal to $L$.
5. Trapping of externally generated ions. - The electrostatic trap is not very favourable for the purpose of trapping ions injected from an external source. This is due to the static nature of the confining field configuration and to the inverse proportionality relationship of the radial restoring force and the radial displacement. A numerical analysis was carried out in detail to investigate the properties of the electrostatic trap for external ion injection. Hereby ion orbits were calculated for a singly charged ion of a mass number of 100 and an energy of $20 \mathrm{eV}$ injected into an electrostatic trap with $R_{2}=1.25 \mathrm{~cm}$ and $R_{1}=0.01 \mathrm{~cm}$ operated at a potential difference of $1000 \mathrm{~V}$. When the injected beam is at $45^{\circ}$ measured from a radius vector, the time the ion is inside the trap is just $1.8 \mu \mathrm{s}$. The injected ion goes along a curving orbit to the other side of the outer cylinder and is lost, as depicted in figure $6 a$. However the trapping time for some of the ions can be improved by superimposing an oscillating voltage to the constant potential difference. The purpose of the modulating field is to modify the ion orbit slightly so that for a finite period of time the ion can be clear of both of the two cylinders. Examples of the modified orbit are shown in figures $6 b$ and $6 c$. A plot of the trapping time versus the injection phase measured with respect to the modulation field is depicted in figure 7 for a modulation amplitude of $100 \mathrm{~V}$ and a frequency of $2 \mathrm{MHz}$. For a limited portion of the modulation period the trapping time is found to exceed $50 \mu \mathrm{s}$.
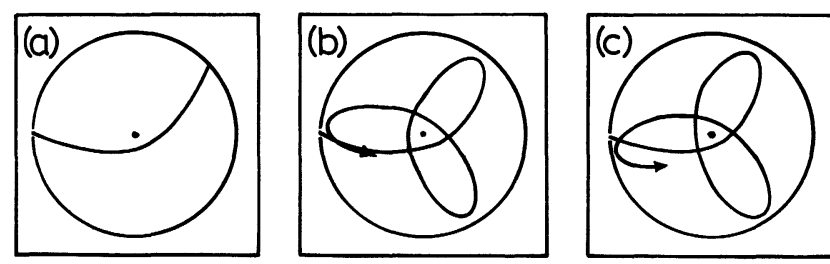

Fig. 6. - Ion trajectories on a radial plane for a singly charged ion $(m=100 \mathrm{u}, E=20 \mathrm{eV})$ injected into an electrostatic $\operatorname{trap}\left(R_{1}=0.01 \mathrm{~cm}\right.$, $R_{2}=1.25 \mathrm{~cm}$, and $V=1000 \mathrm{~V}$ ). (a) No modulation; (b) with modulation field : $100 \mathrm{~V}$ and $2 \mathrm{MHz}$, and $(c)$ with modulation field : $100 \mathrm{~V}$ and $1 \mathrm{MHz}$.

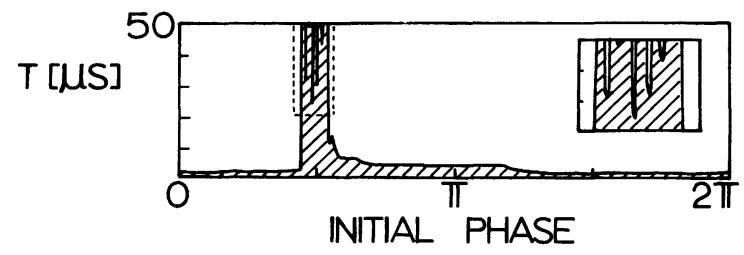

Fig. 7. - Trapping time versus the injection phase measured with respect to the modulation field for the same case as figure $6 b$. The portion of the curve inside the dash line is also shown on an expanded initial phase scale in the insert.

6. Conclusions. - In principle ion confinement is possible for indefinite times in an electrostatic trap. Ions of very different values of the charge-to-mass 
ratio can be confined simultaneously. The trapped ions spend most of their time orbiting in the region between the two cylinders where the electric field strength is low so that Stark splitting is not serious. Because of the inverse proportional relation between the radial displacement and the electric attractive force, the electrostatic trap is not expected to be suitable for trapping externally injected ions. However the addition of a superimposed modulating field can improve its confinement property for externally generated ions.

The presence of ions is monitored by observing the fluorescent light when a laser is tuned to resonance with the ion resonance lines. This non-destructive ion detection method is preferred over the pulsed detection scheme, where at the end of the observation cycle the trapping voltage polarity is reversed and the ion number is counted by a particle detector. It is also pointed out that resonance methods like damping of a tuned circuit or monitoring the coherent or incoherent ion oscillation are not practical because of the continuous range of frequencies present and the resulting small power density per frequency interval. However, if a magnetic field of suitable field strength can be superimposed along the cylinder axis all ions with the same $q / m$ will acquire the same cyclotron frequency. In such a case ion specific monitoring and resonance methods can be used by selectively exciting the cyclotron motion and observing the induced voltage in a split external cylinder ion trap.

Acknowledgments. - This work is supported by the U.S. National Science Foundation under Grant Number Phy 81-11943 and by the U.S. Department of Energy under Contract Number DE-AS05-80 ER 10578.

\section{References}

[1] Schuessler, H. A., in Progress in Atomic Spectroscopy, Hanle, W. and Kleinpoppen, H., eds. (Plenum) 1978, pp. 999.

[2] Dehmelt, H. G., in Atomic Physics 7, Kleppner, D. and Pipkin, F., eds. (Plenum) 1981.

[3] Schuessler, H. A. in Physics of Atoms and Molecules, Coherence and Correlations in Atomic Collisions, Kleinpoppen, H., and Williams, J. F., eds. (Plenum) 1980, pp. 423

[4] Galiouna Nassiopoulos, A., Möller, P. A., Septier, A., Revue Phys. Appl. 15 (1980) 1529.

[5] Rettinghaus, G., Z. Angew. Phys. 22 (1967) 321.

[6] Fischer, E., Z. Phys. 156 (1959) 1.

[7] Penning, F. M., Physica 3 (1936) 873.

[8] O Chun-Sing, Schuessler, H. A., J. Appl. Phys. (April 1981).

[9] Kingdon, K. H., Phys. Rev. 121 (1923) 408.
[10] Hooverman, R. H., J. Appl. Phys. 34 (1963) 3505.

[11] Dehmelt, H. G., Adv. At. Mol. Phys. 3 (1967) 53 ; 5 (1969) 109.

[12] Prior, M. H., Wang, E. C., Phys. Rev. 16 (1977) 6.

[13] Vane, C. R., Prior, M. H., Marrus, R., Phys. Rev. Lett. 46 (1981) 107.

[14] O Chun-Sing, Schuessler, H. A., J. Appl. Phys. (March 1981).

[15] O Chun-Sing, Schuessler, H. A., J. Phys. D 14 (1981).

[16] Gray, T. J., Cocke, C. L., Justimano, E., Phys. Rev. A 22 (1980) 849.

[17] Neuhauser, W., Hohenstatt, M., Toschek, P., Dehmelt, H. G., Phys. Rev. Lett. 41 (1978) 233.

[18] Wineland, D. J., Drullinger, R. E., Walls, F. L., Phys. Rev. Lett. 40 (1978) 1639. 